\title{
Magnetic Behavior of Europium Silicates Using Nuclear Gamma Resonance Spectroscopy *
}

\author{
G. M. Kalvius and G. K. Shenoy \\ Argonne National Laboratory, Argonne, Illinois, and \\ Physik Department, Technische Universität München, Munich, Germany
}

(Z. Naturforsch. 26 a, 353-356 [1971] ; received 9 January 1971)

Dedicated to Prof. Dr. H. Maier-Leibnitz on the occasion of his 60th birthday

\begin{abstract}
The ferromagnetic transition temperature, the quadrupole interaction, the hyperfine magnetic field, and the isomer shift have been measured in $\mathrm{Eu}_{2} \mathrm{SiO}_{4}$ and $\mathrm{Eu}_{3} \mathrm{SiO}_{5}$ using the $21.6 \mathrm{keV}$ nuclear gamma resonance in ${ }^{151} \mathrm{Eu}$. The isomer shifts found $\left(-0.6 \mathrm{~mm} / \mathrm{sec}\right.$ for $\mathrm{Eu}_{2} \mathrm{SiO}_{4}$ and $+0.1 \mathrm{~mm} / \mathrm{sec}$ for $\mathrm{Eu}_{3} \mathrm{SiO}_{5}$ against $\mathrm{EuS}$ ) are less negative than the values typical for other ionic $\mathrm{Eu}^{2+}$ compounds. The magnetic hyperfine fields $\left(280 \mathrm{kOe}\right.$ for $\mathrm{Eu}_{2} \mathrm{SiO}_{4}$ and $240 \mathrm{kOe}$ for $\left.\mathrm{Eu}_{3} \mathrm{SiO}_{5}\right)$ are much lower than the $\mathrm{Eu}^{2+}$ free ion value. A brief qualitative discussion of these hyperfine parameters is given. A rough correlation between the isomer shift and the transition temperature for various divalent Eu compounds is indicated. The effect of trivalent Eu impurities on lowering the Curie temperature of the silicates is demonstrated.
\end{abstract}

\section{Introduction}

The silicates of europium have been investigated recently for their structural, optical, magnetic, and luminescent properties ${ }^{1-4}$. Amongst the known stable phases of the $\left(\mathrm{EuO}-\mathrm{SiO}_{2}\right)$ system, the orthosilicate $\mathrm{Eu}_{2} \mathrm{SiO}_{4}$ and $\mathrm{Eu}_{3} \mathrm{SiO}_{5}$ exhibit ferromagnetic behavior inspite of being ionic transparent insulators ${ }^{1}$. They have thus stirred considerable interest. Nuclear gamma resonance (NGR) is a powerful tool to probe the microscopic behavior of magnetic materials. In particular the $21.6 \mathrm{keV}$ resonance in ${ }^{151} \mathrm{Eu}$ has excellent characteristics for investigating both the magnetic and chemical nature of europium ions. In this contribution we report NGR measurements of the hyperfine parameters in these silicates over the temperature region from $77 \mathrm{~K}$ to $1.5 \mathrm{~K}$. From this we deduce the ferromagnetic transition temperature, the low temperature hyperfine magnetic field, and the isomer shift. A brief qualitative discussion of the findings is given.

\section{Crystal Structure}

Both these silicates are structurally complex. $\mathrm{Eu}_{2} \mathrm{SiO}_{4}$ has either orthorhombic ${ }^{2}$ or monoclinic ${ }^{3}$

Reprints request to Prof. Dr. G. M. Kalvius, PhysikDepartment E 15 der Technischen Universität München, D-8000 München 2, Arcisstraße 21.

* Work supported in part by the U.S. Atomic Energy Commission.

1 M. W. Shafer, T. R. McGuire, and J. C. Suits, Phys. Rev. Letters 11, 251 [1963]. structure depending on the method of synthesis. However, in pure crystals only the monoclinic phase is stable ${ }^{3}$ at temperatures below $165^{\circ} \mathrm{C}$. A small impurity of $\mathrm{Eu}^{3+}$ seems to stabilize the orthorhombic phase at room temperature, but nothing is known about the temperature region below $77 \mathrm{~K}$ where our measurements were performed. In either structure the $\mathrm{Eu}^{2+}$ ion experiences a hexacoordination of oxygen ions, each of which belongs to a different $\mathrm{SiO}_{4}$ tetrahedron.

$\mathrm{Eu}_{3} \mathrm{SiO}_{5}$ belongs to the tetragonal system with space group I4c2. The $\mathrm{Eu}^{2+}$ ions have two site symmetries. While at both the sites the $\mathrm{Eu}^{2+}$ ions are surrounded by distorted oxygen octahedra; the nearest and next nearest oxygen distances differ slightly for the two sites.

\section{Experimental}

Conventional experimental techniques of NGR spectroscopy have been adopted in the present investigation. All measurements were performed in transmission geometry with a $50 \mathrm{mCi}$ source of ${ }^{151} \mathrm{Sm}_{2} \mathrm{O}_{3}$. The source was always kept at roughly the same temperature as the absorber. All samples investigated have been obtained from IBM-Laboratories where the compounds have been synthesized by solid-state reaction of $\mathrm{EuO}$

2 R. C. Rau, Rare Earth Research II, Proc. Third Rare Earth Conf., Florida 1963. Ed. K. S. VoveEs, Gordon and Breach Publ. Co., New York 1964, and Acta Cryst. 17, 1483 [1964].

3 G. Busch, E. Kaldis, R. Verreault, and H. Felsche, Materials Res. Bull. 5, 17 [1970]. - E. Kaldis, P. Streit, and P. Wachter, J. Phys. Chem. Solids (to be published).

4 J. E. Weidenborner, N.R. Stemple, and Y. Okaya, Proc. Amer. Cryst. Association, Gatlinburg, Tenn., 1965, p. 69. 
and $\mathrm{SiO}_{2}$ in a hydrogen atmosphere ${ }^{1}$. The resulting crystals were crushed by us into fine powder and absorbers of about $5-10 \mathrm{mg} / \mathrm{cm}^{2}$ thickness were formed by spreading the powder into a disc-shaped lucite container. This then was sealed in an inert and dry atmosphere.

The NGR spectra were analyzed on an IBM 360/75 computer using a least-squares fitting routine discussed earlier ${ }^{5}$.

All isomer shifts are given with reference to a standard EuS absorber ${ }^{6}$.

\section{Results}

The resonance spectra of both silicates at $77 \mathrm{~K}$ showed a strong absorption centered around $-0.5 \mathrm{~mm} / \mathrm{sec}$. This isomer shift (IS) is representative of a divalent europium ion ${ }^{7}$. The width of this absorption peak is broader than that obtained with an equally thick paramagnetic EuS absorber using the same source. Since the $\mathrm{Eu}^{2+}$ ions in both silicates have a low site symmetry, the observed broadening is considered to be due to quadrupole interactions. Unfortunately the ratio of the nuclear quadrupole moments of the ground state and the $21.6 \mathrm{keV}$ excited state is such that pure quadrupole spectra are commonly unresolved for this resonance ${ }^{5}$. The computer analysis gives an interaction energy of $e^{2} q Q=-12 \mathrm{~mm} / \mathrm{sec}$ in $\mathrm{Eu}_{2} \mathrm{SiO}_{4}$. A somewhat larger value was obtained for $\mathrm{Eu}_{3} \mathrm{SiO}_{5}$. However, in this compound $\mathrm{Eu}^{2+}$ ions occupy two sites with different symmetries and one actually should fit two different quadrupole spectra to the experimental spectrum. In view of the poor resolution, this was not attempted and only the average value for $e^{2} q Q$ was derived. The results are given in Table 1.
In addition to the $\mathrm{Eu}^{2+}$ absorption a weak resonance is found near $+13.3 \mathrm{~mm} / \mathrm{sec}$. This corresponds to a trivalent europium impurity present in these compounds. In accordance with Ref. ${ }^{3}$ this is to be expected by the method of preparation used. Because of the large differences in the IS between $\mathrm{Eu}^{2+}$ and $\mathrm{Eu}^{3+}$ ions, the NGR of ${ }^{151} \mathrm{Eu}$ provides a unique non-destructive method to estimate the $\mathrm{Eu}^{3+}$ content in $\mathrm{Eu}^{2+}$ compounds. Assuming the DebyeWaller factor (which gives the strength of the reso-

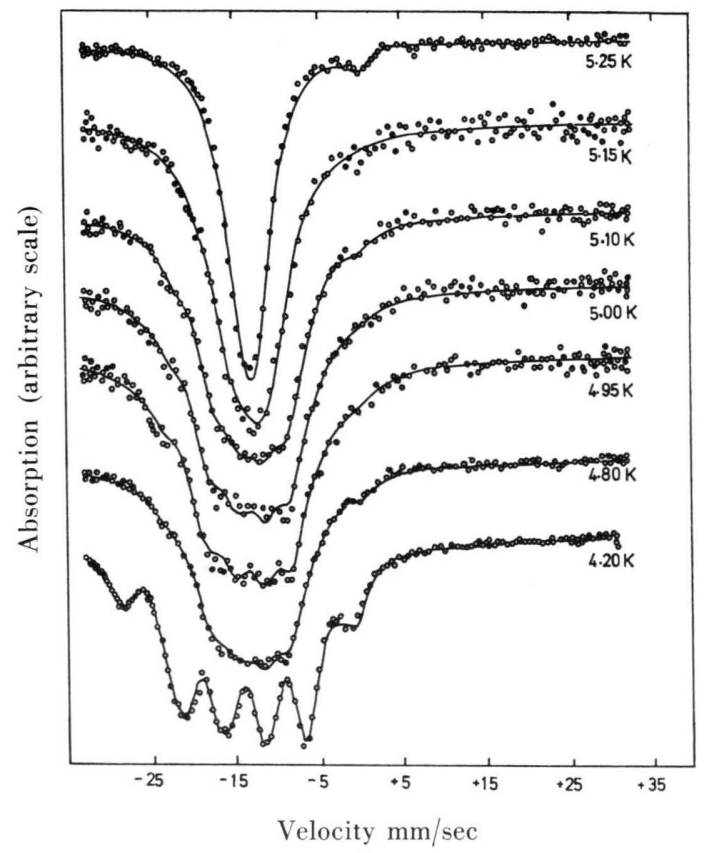

Fig. 1. NGR spectra of $\mathrm{Eu}_{2} \mathrm{SiO}_{4}$ at temperatures close to $T_{\mathrm{c}}(=5.2 \pm 0.5 \mathrm{~K})$. The solid lines are least squares fits as discussed in the text.

Table 1. Curie temperatures and hyperfine interaction parameters for Eu Silicates.

\begin{tabular}{|c|c|c|c|c|c|c|}
\hline Compound & $\begin{array}{c}T_{\mathrm{c}} \\
\text { (pure } \\
\text { sample) } \\
\mathrm{K}\end{array}$ & $\begin{array}{c}T_{\mathrm{c}} \\
\text { (our } \\
\text { sample) } \\
\mathrm{K}\end{array}$ & $\begin{array}{c}\mathrm{Eu}^{3+} \\
(\text { our } \\
\text { sample) } \\
\mathscr{\%}\end{array}$ & $\begin{array}{c}e^{2} q Q \\
\mathrm{~mm} / \mathrm{sec}\end{array}$ & $\begin{array}{l}H_{\mathrm{hf}} \\
\mathrm{kOe}\end{array}$ & $\begin{array}{c}\text { Isomer shift } \\
\text { (vers. EuS) } \\
\text { mm/sec }\end{array}$ \\
\hline $\begin{array}{l}\mathrm{Eu}_{2} \mathrm{SiO}_{4} \\
\mathrm{Eu}_{3} \mathrm{SiO}_{5} \\
\mathrm{Eu}_{3} \mathrm{SiO}_{5}\end{array}$ & $\begin{array}{r}9 \mathrm{a} \\
19 \mathrm{~b}\end{array}$ & $\begin{array}{r}5.2 \pm 0.5 \\
11.5 \pm 0.5\end{array}$ & $\begin{array}{l}5 \\
8\end{array}$ & $\begin{array}{l}-12.0 \pm 0.3 \mathrm{c} \\
-19.0 \pm 10 \mathrm{c}, \mathrm{d}\end{array}$ & $\begin{array}{l}280 \pm 5 \text { e } \\
240 \pm 10 \text { e, d }\end{array}$ & $\begin{array}{l}-0.6 \pm 0.1 \\
+0.1 \pm 0.15\end{array}$ \\
\hline
\end{tabular}

a From Ref. ${ }^{3}$; b from Ref. ${ }^{12}$; c from spectrum taken at $77 \mathrm{~K}$, d average value for both lattice sites;

e from spectrum at $1.6 \mathrm{~K}$.

5 G. M. Kalvius, G. K. Shenoy, G. J. Ehnholm, T. E. Katila, O. V. Lounasmaa, and P. Reivari, Phys. Rev. 187, 1503 [1969], and references given therein.
6 R. L. Cohen and G. M. Kalvius, Nucl. Instr. Methods 86, 209 [1970].

7 G. Gerth, P. Kienle, and K. Luchner, Phys. Letters 27 A, 557 [1968]. 
nance absorption) to be the same for both the impurity $\mathrm{Eu}^{3+}$ and the host $\mathrm{Eu}^{2+}$ ions, we have estimated the $\mathrm{Eu}^{3+}$ content in our samples. The results are also given in Table 1 .

Both $\mathrm{Eu}_{2} \mathrm{SiO}_{4}$ and $\mathrm{Eu}_{3} \mathrm{SiO}_{5}$ are known to be ferromagnetic ${ }^{1}$ at $4.2 \mathrm{~K}$. The transition temperatures are reported ${ }^{4}$ to be $7 \mathrm{~K}$ and $19 \mathrm{~K}$, respectively. The NGR spectra measured well below the Curie temperature $\left(T_{\mathrm{c}}\right)$ showed a well developed hyperfine (hf) magnetic splitting. Close to the transition temperature the hf magnetic field $\left(H_{\mathrm{hf}}\right)$ decreases rather rapidly and becomes zero at $T_{\mathrm{c}}$. In Fig. 1 we show spectra taken around $T_{\mathrm{c}}$ for $\mathrm{Eu}_{2} \mathrm{SiO}_{4}$. By plotting $H_{\mathrm{hf}}$ versus $T$ and extrapolating for $H_{\mathrm{hf}}=0$, we find $T_{\mathrm{c}}$ to be $(5.2 \pm 0.5) \mathrm{K}$ for $\mathrm{Eu}_{2} \mathrm{SiO}_{4}$ and $(11.5 \pm 0.5) \mathrm{K}$ for $\mathrm{Eu}_{3} \mathrm{SiO}_{5}$.

Resonance spectra measured at $1.6 \mathrm{~K}$ were analyzed using a Hamiltonian for an axially symmetrie quadrupole interaction $(\eta=0)$ and with the direction of $H_{\mathrm{hf}}$ along the principal efg axis $(\Theta=0$, $\Phi=0)$. Because of the low symmetry of the $\mathrm{Eu}^{2+}$ ions in the silicates these assumptions are surely an oversimplification. On the other hand, a fit with the correct Hamiltonian contains too many free variables and the fitting routine is unable to find a reproducible set of minimized parameters. Using a simulation program for the calculation of theoretical hf spectra we have shown earlier ${ }^{5}$ that the structure of the spectrum is not sensitive to the chosen values of $\Theta$ and $\Phi$, provided $g H_{\mathrm{hf}} \gg e^{2} q Q / 4 I(2 I-1)$.

Furthermore, under this condition, the influence of $\eta$ is barely noticable, in particular for values of $\eta<0.4$. From these simulations it is seen that using the simple Hamiltonian described earlier, the deduced value of $H_{\text {hf }}$ is correct within the experimental limits of error. But the number for $e^{2} q Q$ returned by the fit is in general smaller than its actual value. This effect is clearly seen in our results, which give a value of $e^{2} q Q=-4.3 \mathrm{~mm} / \mathrm{sec}$ from the fitting of the magnetic spectrum of $\mathrm{Eu}_{2} \mathrm{SiO}_{4}$. This number is about $30 \%$ of the value deduced from the pure quadrupole spectrum (Table 1 ). We take this as evidence that $H_{\mathrm{hf}}$ makes an angle with the principal efg axis. The spectrum of $\mathrm{Eu}_{3} \mathrm{SiO}_{5}$ did not show this effect quite so pronounced, but its analysis is further complicated by the presence of two lattice sites for $\mathrm{Eu}^{2+}$. However, the spectra taken well below $T_{\mathrm{c}}$

8 T. KASUYA, Symposium on Magnetic Semiconductors, IBM-Journal of Research and Development 14, 214 [1970]. clearly show that the magnetic hf fields must be nearly the same for both sites. The value for $H_{\mathrm{hf}}$ given in Table 1 is an average. From the observed line width we can set a limit of $10 \%$ for the difference in $H_{\mathrm{hf}}$ for the two sites. No difference in IS within the experimental errors was found for the resonances above and below the Curie points.

\section{Discussion}

The nature of the exchange interaction in divalent europium compounds has at present been discussed mainly for the chalcogenides ${ }^{8}$. The molecular field model is used in deriving the coupling between the nearest neighbors $\left(J_{1}\right)$ and the next nearest neighbors $\left(J_{2}\right)$. The relative strength of $J_{1}$ compared to $J_{2}$ determines the basic magnetic behavior since $J_{1}$ is a ferromagnetic coupling while $J_{2}$ is antiferromagnetic. A 4f-5d hybridization leads to an overlap of the orbitals of nearest neighbors and produces $J_{1}$. The antiferromagnetic coupling is due to superexchange via the $p$ orbitals of the anions. Thus ferromagnetism $\left(J_{1} \gg J_{2}\right)$ is observed if the energy of promoting $4 \mathrm{f}$ electrons into $5 \mathrm{~d}$ orbitals is small and if the $5 \mathrm{~d}-4 \mathrm{f}$ overlap is large. The first condition is mainly governed by the lattice parameter, the second by the symmetry of the anion field. In the silicates the $\mathrm{Eu}^{2+}$ are surrounded by a distorted oxygen octahedron which presumably puts the $t_{2 g}-5 d$ orbitals lower in energy than the $e_{g}-5 d$ orbitals. A simple minded comparision between the chalcogenides and the silicates thus predicts a rather high $T_{\mathrm{c}}$ for the latter ${ }^{4}$. However, the lowering of octahedral symmetry (distortion) will lift the degeneracy of $t_{2 g}$ orbitals, which in turn will reduce the $5 \mathrm{~d}-4 \mathrm{f}$ overlap and thereby lower the transition temperature.

The electronic structure of the cation $\left(\mathrm{Eu}^{2+}\right)$ is reflected in the observed IS. From the above discussion it is obvious that there should be some relation between the IS, the transition temperature, and the type of ordering. We have plotted in Fig. 2 the IS of non-metallic compounds of $\mathrm{Eu}^{2+}$ versus the transition temperature as they are reported in the literature ${ }^{9}$. From this it can be seen that the $\mathrm{Eu}^{2+}$ compounds with higher transition temperature tend to show a higher s-electron density at the nucleus.

9 G. J. Ehnholm, T. E. Katila, O. V. Lounasmaa, R. Reivari, G. M. Kalvius, and G. K. Shenoy, Z. Physik 235, 289 [1970], and references given therein. 


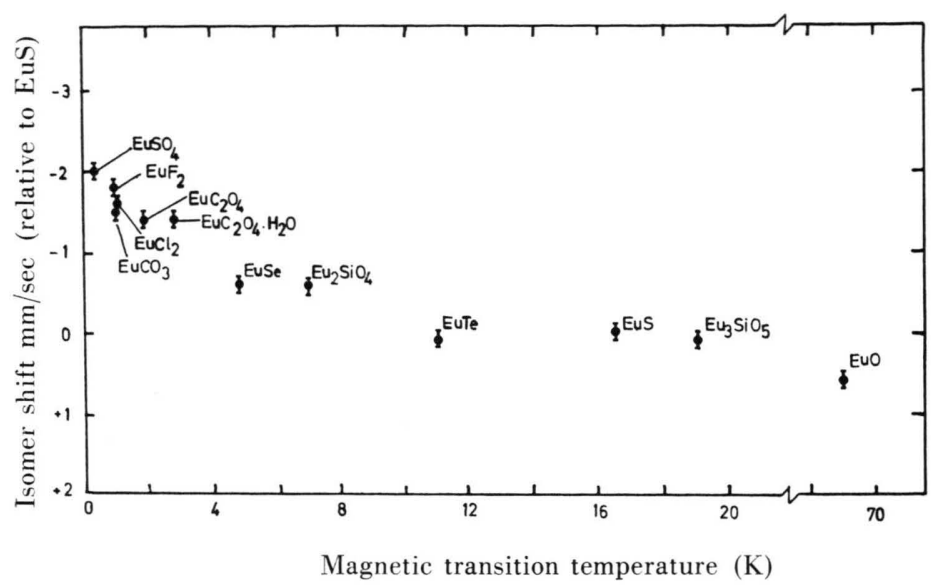

Fig. 2. Isomer shift of various non-metallic $\mathrm{Eu}^{2+}$ compounds plotted against their magnetic transition temperatures. Most of the data are taken from Ref. ${ }^{9}$.

The antiferromagnetic ionic compounds like $\mathrm{EuSO}_{4}$ are expected to come closest to a pure $4 \mathrm{f}^{7}$ configuration. The promotion of $4 \mathrm{f}$ electrons into $5 \mathrm{~d}$ orbitals will increase the charge density at the nucleus, since the shielding of $6 \mathrm{~s}$ electrons by $5 \mathrm{~d}$ electrons is only about $30 \%$ of that by $4 \mathrm{f}$ electrons. Using a HartreeFock self-consistent field calculation ${ }^{10}$ it should be posible to estimate the amount of $5 \mathrm{~d}$ hybridization. However, we have refrained from doing so, because the effect of increasing $6 \mathrm{~s}$ overlap with decreasing lattice parameter will also markedly influence the IS. Both these contributions are inseparable. The silicates in question show IS which are in general agree-

10 G. K. Shenoy and G. M. Kalvius, unpublished.

11 J. M. Baker and F. I. B. Williams, Proc. Roy. Soc. London A 267, 283 [1962]. ment with this qualitative picture. Although the silicates are usually termed "ionic compounds", it should be noted that their IS are less negative than the ones of the more typical ionic compounds (like $\mathrm{EuSO}_{4}$ and $\mathrm{EuF}_{2}$ ) and more in the region occupied by the chalcogenides. In addition, the hf fields found in both silicates are markedly lower than the value reported ${ }^{11}$ for the free $\mathrm{Eu}^{2+}$ ion $(-341 \mathrm{kOe})$. In contrast, the hyperfine fields of the ionic $\mathrm{Eu}^{2+}$ compounds $\left(\mathrm{EuSO}_{4}, \mathrm{EuF}_{2}\right.$ etc.) are all rather close to the free ion number ${ }^{9}$. In the silicates an additional positive field, produced either by the dipolar contribution or by the $5 \mathrm{~d}$ electrons, thus reduces the observed $H_{\mathrm{hf}}$.

The values for $T_{\mathrm{c}}$ reported here are somewhat lower than those given by KaLdis et al. ${ }^{3}$ and SHAFER ${ }^{12}$. This lowering of $T_{\mathrm{c}}$ can be attributed to the magnetic dilution of our samples by the non-magnetic $\mathrm{Eu}^{3+}\left({ }^{6} \mathrm{~F}_{0}\right)$ impurity ions. The estimated $\mathrm{Eu}^{3+}$ content of our samples is $5 \%$ in $\mathrm{Eu}_{2} \mathrm{SiO}_{4}$ and $8 \%$ in $\mathrm{Eu}_{3} \mathrm{SiO}_{5}$ (see Table 1). This finding is in agreement with an earlier report by McGuire and SHAFER ${ }^{13}$, who give $T_{\mathrm{c}}=4 \mathrm{~K}$ for a sample of $\mathrm{Eu}_{3} \mathrm{SiO}_{5}$ containing roughly $20 \% \mathrm{Eu}^{3+}$ impurities.

\section{Acknowledgement}

We thank Drs. Petrich and Shafer of the IBM Laboratory for providing us with the samples. Dr. WACHTER (ETH Zurich) was kind enough to send us a copy of his paper prior to publication.

12 M. W. Shafer, J. Appl. Phys. 36, 1145 [1965].

13 T. R. McGuire and M. W. Shafer, J. Appl. Phys. 35, 984 [1964]. 